\title{
THE BUTCHER-OEMLER EFFECT IN NEARBY CLUSTERS
}

\author{
R.M. SHARPLES \\ University of Durham \\ Department of Physics, South Road, Durham, UK.
}

\section{Introduction}

Probably the most striking evidence for galaxy evolution at recent epochs has been the discovery of a rapid change in the nature of galaxy populations in clusters over the redshift range $\mathrm{z}=0$ to $\mathrm{z}=0.5$. The 'classical' ButcherOemler effect (Butcher \& Oemler 1978, 1984) used photomteric studies to reveal an unexpected increase in the fraction of blue galaxies in the cores of distant $(\mathrm{z} \sim 0.4)$ rich concentrated clusters when compared with nearby $(\mathrm{z}<0.05)$ clusters of similarly high richness and central concentration. An alternative view, based on spectroscopic studies (Dressler \& Gunn 1982; Couch \& Sharples 1987), manifests itself as an increase in the fraction of active galaxies which show signs of recent star formation and/or nuclear activity. Some of these galaxies are indeed blue but some (e.g. in Cl0016+16, Dressler \& Gunn 1992) are red. Although it is the very absence of blue galaxies in nearby clusters which defines the classical Butcher-Oemler effect, comparable spectroscopic studies of nearby cluster populations with the appropriate completeness and high signal-to-noise required for population (as opposed to dynamical) studies have only recently been undertaken. In at least one case these have revealed unexpected similarities to the spectroscopic signatures which appear so prevalent at higher redshifts.

\section{Environmental Effects on E/SO Galaxies in Nearby Clusters}

Early-type (E/S0) galaxies are conventionally viewed as old stellar systems which formed the bulk of their stars in the first 1-2 Gyrs after their formation epoch. However, there is now a growing body of evidence to suggest that recent star formation has occured in at least some nearby E/S0 galaxies (O'Connell 1980; Rose 1985, Davies 1995). Bower et al. (1990) used gravity-sensitive spectral indices in the blue spectral region to show that star formation in early-type galaxies in the cores of nearby rich clusters was 
truncated at an earlier epoch (on average) than similar galaxies in the field. A important handle on the question of whether such differences are primarily the result of nature (formation mechanism) or nurture (environmental effects) can be obtained by studying the early-type galaxy population in the outer parts of rich clusters where the environment is intermediate between the dense core regions and that of the field.

\section{Star Formation in Early-Type Galaxies in the Coma Cluster}

Some years ago we began such a study of the Coma cluster with the goal of studying distribution of $\mathrm{E} / \mathrm{S} 0$ galaxies in the $\mathrm{SrII} / \mathrm{H} \delta / \mathrm{FeI}$ plane (Rose 1985 ) at a projected distance of $\mathrm{R} \sim 1 h^{-1} \mathrm{Mpc}$ from the cluster centre. Since no deep wide-area morphological classification surveys were available, the sample was selected primarily by isolating early-type galaxies using the colour-magnitude sequence and subsequently rejecting any objects which showed evidence of spiral structure. Spectra have now been obtained for 184 galaxies in 3 fields.

\subsection{SPATIAL DISTRIBUTION}

The surprising result to emerge from the first two fields analysed by Caldwell et al. (1993) was that a significant fraction (20/125) of the objects studied had abnormal spectra for early-type galaxies, exhibiting either emission-lines or strong Balmer absorption lines similar to the so-called 'post-starburst' galaxies (Dressler \& Gunn 1982) in the distant ButcherOemler clusters. The great majority of these unusual spectra $(15 / 20)$ occured in galaxies located in the SW field but, since only 2 of the 3 fields could be observed on this first run due to weather, it was unclear whether this effect was a real radial gradient in the galaxy population of the cluster or due to some special conditions in the SW field. We have now obtained spectra for galaxies in the NE field and the fraction of abnormal spectra is again low (similar to that for the central field) thus confirming that the effect cannot be primarily due to a radial gradient (see Fig. 1)

The first indications of what might give rise to the peculiar nature of the SW field came with the publication of the ROSAT wide-field X-ray map which showed a secondary peak of X-ray emission, probably associated with a group of galaxies surrounding the cD galaxy NGC 4839 which lies near the centre of the SW field. This association fits nicely with a picture in which the star formation activity in the Coma galaxies (and by implication the activity seen in higher redshift clusters) was triggered by the interaction of a bound subclump of galaxies with the main cluster. More detailed analysis of the velocity distribution and the X-ray morphology (Burns et al. 1994) in fact favours a model in which the subclump has already passed through 


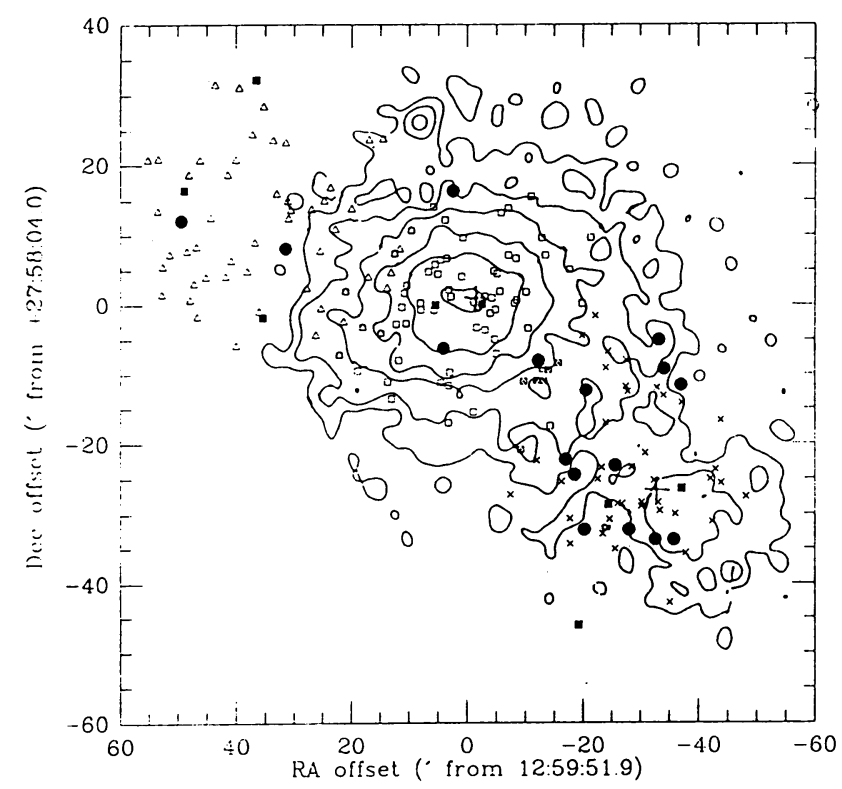

Figure 1. Spatial distribution of Coma E/S0 galaxies overlaid on a ROSAT X-ray map from Briel et al (1992). Spectra have been obtained in three 40 arcmin diameter fields using the Hydra fibre spectrograph at KPNO. Normal spectrum galaxies are denoted by open symbols, abnormal galaxies by filled symbols.

the cluster core where the effects of the dense intra-cluster medium (Evrard 1991) or frequent galaxy interactions (Moore 1995) may have triggered the recent star formation activity.

\subsection{SPECTRAL ANALYSIS}

The majority of the abnormal spectra in the SW clump exhibit strong Balmer lines in addition to the usual metallic features (CaII, G band, Mgb) expected for an old stellar population. Their spectra are reminiscent of the ' $\mathrm{E}+\mathrm{A}$ ' galaxies found in abundance in the cores of distant Butcher-Oemler clusters but with generally redder colours. The most direct analogues are the class of ' $\mathrm{H} \delta$-strong' galaxies identified in three $\mathrm{z}=0.31$ clusters by Couch \& Sharples (1987).

Because the galaxies are relatively nearby, the Coma sample gives us an opportunity to study in unprecedented detail an environmental effect which is probably closely related to the Butcher-Oemler phenomenon. Two of the most obvious questions are when did the star-formation activity which produces the strong Balmer-line objects take place, and was this activity confined to the nuclear regions of the galaxies or was it a global phenomenon? Leonardi \& Rose (1995) present a new technique for accu- 
rately determining the ages of starbursts in post-starburst galaxies which Caldwell et al. (1995) use to show that star-formation bursts in a small subsample of the Coma galaxies ceased 1-1.5 Gyr ago, consistent with the dynamical estimates of the interaction timescale from Burns et al. (1994). The remnant intermediate age stars are found to be distributed over a large range in radius although changes in the fraction of light coming from this population is evident in some cases. Further work on the nature of the Coma post-starburst galaxies is in progress along with comparable studies of other nearby rich clusters which should reveal the extent to which these environmental effects on stellar populations are typical of clusters at the present epoch.

\section{Acknowledgements}

I am grateful to my colleagues Jim Rose, Nelson Caldwell and Richard Ellis for permission to use data in advance of publication and for many useful discussions on the nature of the Butcher-Oemler effect.

\section{References}

Bower, R.G., Ellis, R.S., Rose, J.A. and Sharples, R.M. (1990) $A J$, Vol. no. 99, p. 530 Briel, U.G, Henry, J.P. and Bohringer, H. (1992) $A \mho A$, Vol. no. 259, p. L31

Burns, J.O., Roettiger, K., Ledlow, M. and Klypin, A. (1994) ApJ, Vol. no. 427, p. L87

Butcher, H.R. and Oemler, A. (1978) $A p J$, Vol. no. 219, p. 18

Butcher, H.R. and Oemler, A. (1984) ApJ, Vol. no. 285, p. 426

Caldwell, C.N., Rose, J.A., Franx, M., and Leonardi, A. (1995) Preprint

Caldwell, C.N., Rose, J.A., Sharples, R.M., Ellis, R.S., and Bower, R.G. (1994) AJ, Vol. no. 106, p. 473

Couch, W.J. and Sharples, R.M. (1987) MNRAS, Vol. no. 229, p. 42

Davies, R.L. (1995) in, New light on Galaxy Evolution, IAU 171, p. XXX.

Dressler, A. and Gunn, J.E. (1982) $A p J$, Vol. no. 263, p. 533

Dressler, A. and Gunn, J.E. (1992) ApJSupp, Vol. no. 78, p. 1

Evrard, A.E. (1991) MNRAS, Vol. no. 248, p. 8P

Leonardi, A. and Rose, J.A. (1995) Preprint

Moore, B. (1995) in, New light on Galaxy Evolution, IAU 171, p. XXX.

O'Connell, R. (1980) $A p J$, Vol. no. 236, p. 430

Rose, J.A. (1985) $A J$, Vol. no. 90, p. 1927

\section{DISCUSSION}

LIU: What is the fraction of abnormal galaxies in the SW clump and in Coma as a whole, and how does this compare with the active galaxy fraction in more distant B-O clusters?

SHARPLES: In the SW clump it is $15 / 64$ or $23 \%$. Over all three fields this drops to $14 \%$. In the distant clusters it is $\sim 20 \%$ based on either the blue fraction or the number of post-starburst spectra. 